\title{
DDAG publishes report confirming commitment to equality, diversity and inclusion
}

The Diversity in Dentistry Action Group (DDAG) has published a report entitled Equality, diversity and inclusion within dentistry. A profession wide commitment which has been endorsed by 35 stakeholder organisations within dentistry, to promote collaborative action.

The founding and development of the DDAG was driven by international social justice activity to uphold racial equality. It was recognised that advancing efforts to tackle inequalities would support the best possible outcomes for patients, the profession and broader society.

The ambition of the DDAG is to co-create a systematic approach for practical action across the profession, to nurture a culture of equality and inclusion and to address issues such as discrimination and underrepresentation, with the aim that this should result in positive changes in all areas of dentistry, including education, workforce development, staff wellbeing and patient care.

The DDAG enables organisations to share good practice, opportunities for collaboration and support, and learn from one another. Its inaugural event in November drew over 70 representatives from across the profession.

The newly-published report sets out the DDAG's aims, plans and recommendations. It marks the DDAG's commitment to collaborative action and confirms the dental organisations which have pledged to the key principles for change:

- Working together to address inequalities and increase diversity in dentistry

- Ongoing improvement through constant enquiry, investigation and learning

- Creating a positive open learning culture across the profession and organisations, which develops understanding and supports people to embrace diversity and inclusion

- Exploring and developing opportunities for representation and inclusion

- Actively committing to addressing racism and discrimination
- Organisational commitment to change at all levels

- Embracing workforce diversity.

There will be an initial focus by the DDAG on racial equality in dentistry, with this later expanding to address wider protected characteristics and discrimination issues.

Chair of the DDAG, GDP and Clinical

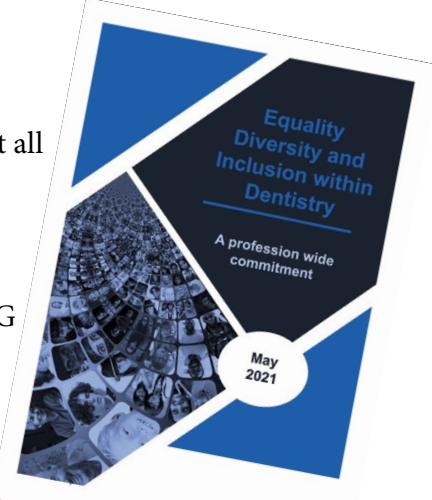
Leadership Manager for the Office of the Chief Dental Officer, Nishma Sharma, said:

'The issue of racism, prejudice, bias is an outdated, stagnant corroded bolus, stuck and wedged into the throat of society; choking progression, suffocating innovation, stifling the potential of a fresh representative workforce where there are no glass ceilings. This can no longer be ignored. Its deep roots are steeped in history, entrenched in the many conflicts and injustices endured over the years. It is now time to break down the barriers using the armoury of education, understanding, empathy, proactive reaction, energy, insight and allyship. Together we are a profession, yes, but we are also individuals with a responsibility for change within our own spheres of influence and extending to wider society'.

The DDAG's report, which is being hosted by the Faculty of General Dental Practice (UK), can be viewed at: https://www.fgdp. org.uk/equality-diversity-and-inclusion.

The FGDP, CGDent and the British Dental Association (BDA) have also established a Racial Equality, Diversity and Inclusion Working Group, to look specifically at disadvantages and inequality of opportunity among ethnic minorities within the dental professions. They are also collaborating on research into experiences of racism, racial discrimination and racial inequalities within the profession, and how these affect individual practitioners.

\section{Study will capture the lived experience of 'everyday' race-related incidents in minority ethnic UK dentists}

As part of a programme of work on the theme of racial inequalities and discrimination in dentistry, the British Dental Association (BDA) is carrying out research to learn more about the lived experiences of minority ethnic dentists.

The BDA is inviting minority ethnic dentists in all spheres of practice, working in the UK, to take part in an online diary study. Participants will be asked to keep an online diary and document any race-related encounters (positive or negative) that happen during their working day over a period of four weeks. It is anticipated that this research will take place May-August 2021, where participants will be able to choose the four-week period convenient to them.

The information provided will allow for a deeper exploration of these everyday race-related encounters and further illuminate the subtler forms of racial prejudice and discrimination and the emotional impact they can have. This research will aid the policy work the BDA will undertake on behalf of the profession.

As a way of saying thank you, participants will receive a $£ 20$ gift card after completing the study.

Anyone who is interested should fill out an expression of interest form, where you will be asked to answer some questions about yourself: https://www.smartsurvey.co.uk/s/ diarystudy_expressionofinterest/.

You do not need to be a member of the BDA to take part. Following this screening process, someone in the BDA's research team will be in contact to let you know if you are eligible to proceed. If you have any questions about the project, please email research@bda.org. 